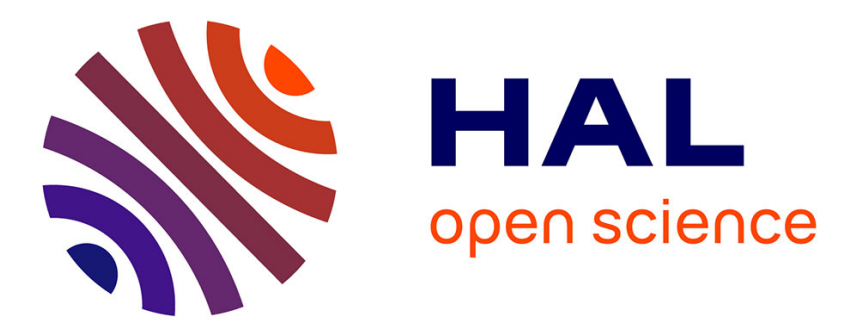

\title{
Un important site mésolithique dans le Nord - Pas-de-Calais : La Mort à Beaurainville
}

Thierry Ducrocq, Sylvie Coutard, Jean-Claude Routier, Anne Bridault

\section{To cite this version:}

Thierry Ducrocq, Sylvie Coutard, Jean-Claude Routier, Anne Bridault. Un important site mésolithique dans le Nord - Pas-de-Calais : La Mort à Beaurainville. Bulletin de la Société préhistorique française, 2013, 110 (1), pp.130 - 133. 10.3406/bspf.2013.14236 . hal-01483890

\section{HAL Id: hal-01483890 \\ https://hal.science/hal-01483890}

Submitted on 20 May 2020

HAL is a multi-disciplinary open access archive for the deposit and dissemination of scientific research documents, whether they are published or not. The documents may come from teaching and research institutions in France or abroad, or from public or private research centers.
L'archive ouverte pluridisciplinaire HAL, est destinée au dépôt et à la diffusion de documents scientifiques de niveau recherche, publiés ou non, émanant des établissements d'enseignement et de recherche français ou étrangers, des laboratoires publics ou privés. 


\title{
Un important site mésolithique dans le Nord - Pas-de-Calais : La Mort à Beaurainville
}

\author{
Thierry Ducrocq, Sylvie Coutard, Jean-Claude Routier et Anne Bridault
}

$\mathrm{D}^{\mathrm{s}}$ ES TRANCHÉES superficielles de décapage ont révélé en 2007 des vestiges mésolithiques qui ont ensuite fait l'objet d'une approche complémentaire sous la forme de la fouille manuelle de quelques mètres carrés dispersés sur plusieurs secteurs. Deux datations ${ }^{14} \mathrm{C}$ obtenues récemment renforcent l'intérêt de ce gisement et motivent cette note.

Le site se trouve dans le fond de vallée de la Canche, à environ $30 \mathrm{~km}$ de l'actuel rivage (fig. 1). À une profondeur d'environ $1 \mathrm{~m}$, les vestiges mésolithiques sont inclus dans un limon hydromorphe scellé par des tourbes franches puis des tufs fluviatiles. Latéralement, des tourbes à bois flottés, des limons et des sables calcaires tuffacés se développent sur une épaisseur d'au moins $4 \mathrm{~m}$ et soulignent l'emplacement d'un paléochenal de la Canche. Les hommes du Mésolithique ont ainsi occupé un replat sur sol sec à proximité immédiate d'un cours d'eau. La remontée du plan d'eau a ensuite permis la fossilisation des niveaux archéologiques. Ce type d'implantation et de préservation est très fréquent dans les vallées tourbeuses du Nord de la France (Ducrocq, 2001).

Le diagnostic historique, un peu trop intrusif, a révélé des vestiges sur une surface de près de $3000 \mathrm{~m}^{2}$ avec au moins un amas de débitage et deux structures de combustion dont une marquée par de la terre rubéfiée, de nombreux silex brûlés et des centaines de débris de coquilles de noisettes carbonisées. Une large surface anthropisée de près de $1000 \mathrm{~m}^{2}$ est soulignée par de nombreux petits vestiges brûlés (silex, os, noisettes et charbons).

Bien qu'exempte de collagène, la faune est bien conservée. Trois mètres carrés fouillés et tamisés près du principal foyer (sondage 1) ont livré vingt-cinq restes parfois brûlés attestant de la prédation du chevreuil, du cerf, du sanglier et de l'aurochs. Quelques os exhumés sur d'autres secteurs mettent de nouveau en évidence le sanglier, le cerf et le chevreuil. Pour l'instant, il n'y a pas de trace de poissons ou d'oiseaux.

La matière première lithique est un silex de la craie accessible en abondance très près du site. L'industrie lithique est très riche sur le sondage 1 (fig. $2, \mathrm{n}^{\text {os }} 1$ à 14 ) avec plus de 2000 objets dont plus d'un millier d'esquilles. Réalisé sur place, le débitage, apparenté au style de Coincy (Rozoy, 1969), est orienté vers l'obtention de lamelles irrégulières extraites par percussion directe à la pierre tendre. Une seule chaine opératoire produit une large palette de modules laminaires (fig. $2, \mathrm{n}^{\text {os }} 3$ à 7). Les pièces retouchées sont très rares sur le sondage 1 , avec seulement quelques microlithes, tous figurés ici (fig. $2, \mathrm{n}^{\text {os }} 8$ à 14). Ces pièces peu typiques comportent des lamelles à dos et des fragments d'armatures pointues. Plusieurs centaines d'artefacts ont été récupérés sur d'autres secteurs du site. Il s'agit toujours d'un débitage du style de Coincy, avec parfois quelques blocs grossièrement débités pour obtenir des éclats tranchants. De rares outils du fonds commun et une seule armature (fig. 2, $\mathrm{n}^{\circ} 15$ ) constituent la totalité de l'outillage. Les microburins sont totalement absents à Beaurainville.

L'importante extension spatiale du site a d'abord été perçue comme le résultat de très fréquentes réoccupations du lieu. De possibles reliques de stratigraphie et des pièces lithiques avec des aspects physiques différents orientaient vers cette interprétation, souvent retenue pour expliquer l'étendue de certains sites mésolithiques (Crombé et al., 2006).

Deux échantillons ont fait l'objet d'une datation. Le premier est une coquille de noisette carbonisée issue du foyer du sondage 1. Elle provient de la base du limon à vestiges. Les artefacts y sont patinés. Le second est un charbon issu d'un test manuel réalisé sur la fenêtre 2 à $20 \mathrm{~m}$ du sondage 1 . Il est inclus dans la partie supérieure du limon à vestiges et il est associé à quelques silex exempts de patine. Les résultats sont respectivement : $8165 \pm 45$ BP (7316 à 7058 cal. BC; Lyon 6984 - SacA 19535) et $8105 \pm 45$ BP (7177 à 7041 cal. BC; Lyon 6985 - SacA 19536). Ils plaident pour une stricte contemporanéité des divers secteurs ou pour un écart chronologique limité entre les différentes fréquentations du lieu. Quoi qu'il en soit, la question d'un éventuel vaste campement est posée.

Ces datations permettent aussi d'affiner l'attribution culturelle. Entre 8200 BP et au moins 7800 BP, le Nord de la France et la Belgique sont occupés par le Mésolithique à feuille de gui, souvent qualifié de "Rhein-MeuseSchelde » (RMS : Gob, 1985). Il est daté et bien attesté dans la vallée de la Somme (Ducrocq, 2001). Des feuilles de gui sont aussi signalées sur l'actuel littoral à Hardelot ou Wimereux (Mariette, 1970). Hormis quelques pièces foliacées remarquables, les assemblages microlithiques de cette époque sont largement dominés par des lamelles à dos parfois tronquées. Ainsi, les armatures de Beaurainville ne choqueraient pas dans une industrie lithique de type RMS A.

Le site de Beaurainville est le premier site mésolithique de la région Nord - Pas-de-Calais avec de la faune bien préservée. Mais son intérêt réside aussi dans sa position chronologique, juste après une rupture majeure dans l'évolution mésolithique régionale entre le Beuronien et le RMS (Ducrocq, 2009) ou, suivant les chercheurs, entre le Mésolithique ancien et moyen (Crombé et al., 2008). C'est une des phases les plus mal documentées du Mésolithique du Nord de la France et de la Belgique. De plus, les sites de cette période sont parfois surprenants avec, par exemple, une vaste étendue et des structures en creux 


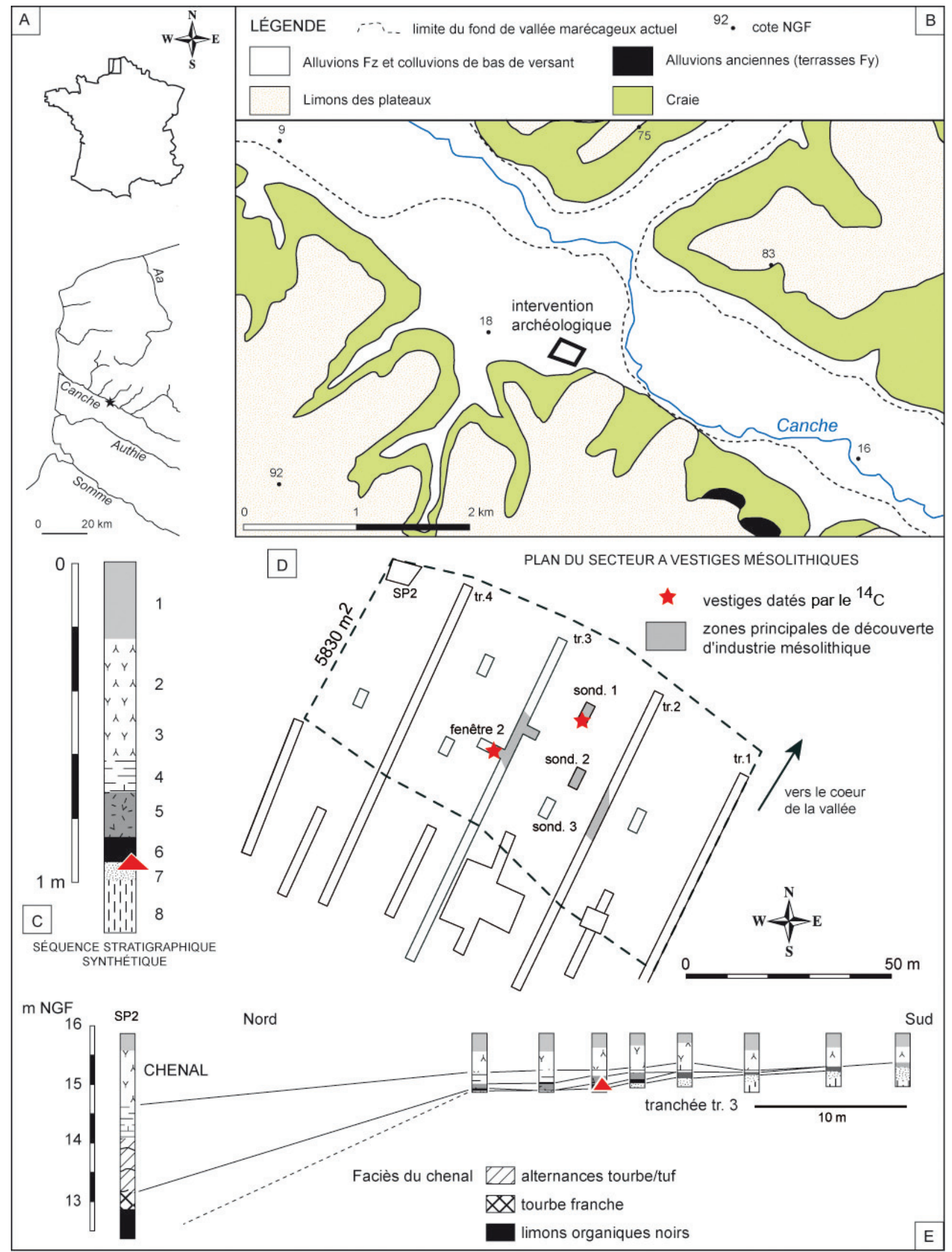

Fig. 1 - La Mort à Beaurainville. A : localisation du site; B : croquis géologique; $\mathrm{C}:$ séquence stratigraphique synthétique $[1:$ terre végétale; 2 : tuf branchu; 3 : tuf granulaire; $4:$ limons calcaires lités; $5:$ mélange tuf/limons organiques noirs; $6:$ limons tourbeux; 7 ; limons gris clair contenant l'industrie mésolithique; $8:$ limons argileux orangés (Bt sur loess)]; $\mathrm{D}:$ plan du secteur à vestiges mésolithiques; E : coupe schématique (document de S. Coutard). 


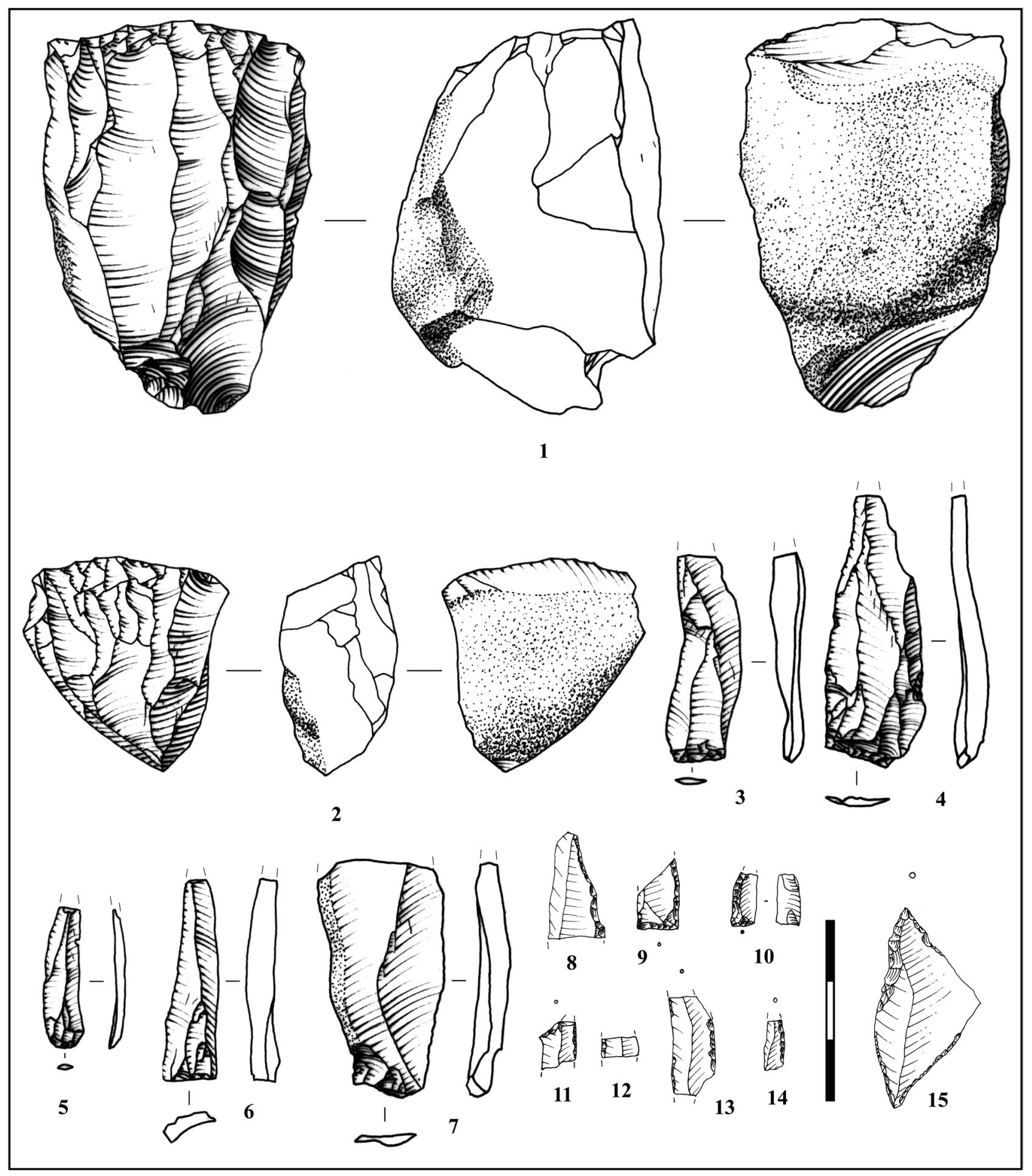

Fig. 2 - La Mort à Beaurainville. 1 à 15 : industrie lithique des sondages $1\left(n^{\text {os }} 1-14\right)$ et $2\left(n^{\circ} 15\right) .1$ et $2:$ nucléus; 3 à $7:$ produits laminaires; 8 à 15 : microlithes (document de $\mathrm{S}$. Coutard; dessins nos 1 à 7 de $\mathrm{S}$. Lancelot et $\mathrm{n}^{\text {os }} 8$ à 15 de T. Ducrocq). 
sur le Petit-Marais de la Chaussée-Tirancourt, ou une certaine anthropisation et des séjours assez longs pour les moments d'occupation les plus anciens de Noyen-surSeine (Mordant et Mordant, 1989; Vigne, 2005), un site peut-être en dehors de la sphère RMS.

Ce nouveau gisement a donc toutes les potentialités pour se prêter à une approche palethnologique qui est un des objectifs majeur des recherches actuelles.

\section{RÉFÉRENCES BIBLIOGRAPHIQUES}

Crombé P., Perdaen Y., Sergant J. (2006) - Extensive Artefact Concentration: Single Occupation or Palimpsests? The Evidence from the Early Mesolithic Site of Verrebroek "Dok" (Belgium), in C. J. Kind (dir.), After the Ice Age. Settlements, Subsistence and Social Development in the Mesolithic of Central Europe, Stuttgart, Konrad Theiss (Materialhefte zur Archäologie in Baden-Württemberg, 78), p. 237-243.

Crombé P., Perdaen Y., Sergant J. (2008) - La transition du Mésolithique ancien au Mésolithique moyen/récent dans le Nord-Ouest de la Belgique : quelques réflexions concernant l'occupation du territoire, in J.-P. Fagnart, A. Thévenin, T. Ducrocq, B. Souffi et P. Coudret (dir.), Le début du Mésolithique en Europe du Nord-Ouest, Paris, Société préhistorique française (Mémoire, 45), p. 195-204.

Ducroce T. (2001) - Le Mésolithique du bassin de la Somme. Insertion dans un cadre morphostratigraphique, environnemental et chronoculturel, Lille, CERP (Publications du CERP, 7), $255 \mathrm{p}$.

Ducroce T. (2009) - Éléments de chronologie absolue du Mésolithique dans le Nord de la France, in P. Crombé, M. Van Strydonck, J. Sergant, M. Boudin et M. Bats (dir.), Chronology and Evolution within the Mesolithic of NorthWest Europe, Newcastle, Cambridge Scholars Publishing, p. 345-362.

Gов A. (1985) - Extension géographique et chronologique de la culture Rhein-Meuse-Schelde (RMS), Helinium, 25, 1, p. 23-36.
Mariette H. (1970) - Préhistoire de la côte d'Opale du Mésolithique à la conquête romaine, Septentrion, 1, p. 90-96.

Mordant C., Mordant D. (1989) - Noyen-sur-Seine, site mésolithique en milieu humide fluviatile, in L'homme et l'eau au temps de la Préhistoire, actes du $112^{\mathrm{e}}$ Congrès national des sociétés savantes (Lyon, 1987), Paris, CTHS, p. 33-52.

Rozoy J.-G. (1969) - Typologie de l'Épipaléolithique (Mésolithique) franco-belge, réunion de 5 separata du Bulletin de la Société préhistorique française (1966, 1967, 1968), Charleville, chez l'auteur, $121 \mathrm{p}$.

Vigne J.-D. (2005) - Découpe du cerf (Cervus elaphus) au Mésolithique moyen, à Noyen-sur-Seine (Seine-et-Marne) : analyses tracéologique et expérimentale, Revue de paléobiologie, volume spécial 10, p. 69-82.

Thierry Ducroce INRAP Nord-Picardie 518, rue Saint-Fuscien, 80000 Amiens thierry.ducrocq@inrap.fr

Sylvie Coutard

UMR 8591 « Laboratoire de géographie physique »

INRAP Nord-Picardie 518, rue Saint-Fuscien, 80000 Amiens sylvie.coutard@inrap.fr

Jean-Claude Routier UMR 8164 « HALMA-IPEL »

INRAP Nord-Picardie

11, rue des Champs, ZI de la Pilaterie, 59650 Villeneuve-d'Ascq jean-claude.routier@inrap.fr

Anne Bridault CNRS, UMR 7041 «ArScAn » Maison de l'archéologie et de l'ethnologie 21, allée de l'Université, 92023 Nanterre cedex anne.bridault@mae.u-paris10.fr 